\title{
Characterizing intonation deficit in motor speech disorders: An autosegmental-metrical analysis of spontaneous speech in hypokinetic dysarthria, ataxic dysarthria and foreign accent syndrome
}

\begin{tabular}{|r|l|}
\hline Journal: & Journal of Speech, Language, and Hearing Research \\
\hline Manuscript ID: & JSLHR-S-11-0263.R2 \\
\hline Manuscript Type: & Research Article \\
\hline Date Submitted by the Author: & n/a \\
\hline Complete List of Authors: & $\begin{array}{l}\text { Lowit, Anja; Strathclyde University, Speech and Language Therapy } \\
\text { Kuschmann, Anja; Strathclyde University, Speech and Language Therapy }\end{array}$ \\
\hline Keywords: & $\begin{array}{l}\text { intonation, autosegmental-metrical (AM) approach, hypokinetic dysarthria, } \\
\text { ataxic dysarthria, foreign accent syndrome }\end{array}$ \\
\hline &
\end{tabular}


Running head: CHARACTERIZING INTONATION DEFICIT IN MSD

Characterizing intonation deficit in motor speech disorders: An autosegmental-metrical analysis of spontaneous speech in hypokinetic dysarthria, ataxic dysarthria and foreign accent syndrome Anja Lowit and Anja Kuschmann University of Strathclyde, Glasgow, UK

\author{
Author note \\ Correspondence concerning this paper should be addressed to Anja Lowit, Strathclyde \\ University, School of Psychological Sciences and Health, Speech and Language Therapy \\ Division, 76 Southbrae Drive, G13 1PP, Glasgow, UK. Email: a.lowit@strath.ac.uk, \\ Phone: +44 (0)141950 3531, Fax: +44 (0)1419503762
}




\title{
CHARACTERIZING INTONATION DEFICIT IN MSD
}

\begin{abstract}
Purpose: The autosegmental-metrical (AM) framework represents an established methodology for intonational analysis in unimpaired speaker populations, but has found little application in describing intonation in motor speech disorders (MSDs). This study compared the intonation patterns of unimpaired participants $(\mathrm{CON})$ and those with Parkinson's Disease (PD), ataxic dysarthria (AT), and foreign accent syndrome (FAS) to evaluate the approach's potential for distinguishing types of motor speech disorders from each other and from unimpaired speech.
\end{abstract}

Method: Spontaneous speech from 8 PD, 8 AT, 4 FAS and 10 CON speakers were analyzed in relation to inventory and prevalence of pitch patterns, accentuation and phrasing. Acousticphonetic baseline measures (maximum-phonation-duration, speech rate and F0-variability) were also performed.

Results: The analyses yielded differences between MSD and CON groups and between the clinical groups regarding prevalence, accentuation and phrasing. AT and FAS speakers used more rising and high pitch accents than PD and CON speakers. The AT group used the highest number of pitch accents per phrase, and all three MSD groups produced significantly shorter phrases than the CON group.

Conclusions: The study succeeded in differentiating MSDs on the basis of intonational performances using the AM approach, thus demonstrating its potential for charting intonational profiles in clinical populations.

Key words: intonation, autosegmental-metrical (AM) approach, hypokinetic dysarthria, ataxic dysarthria, foreign accent syndrome 


\section{CHARACTERIZING INTONATION DEFICIT IN MSD}

Characterizing intonation deficit in motor speech disorders: An autosegmental-metrical analysis of spontaneous speech in hypokinetic dysarthria, ataxic dysarthria and foreign accent syndrome

Prosodic disturbances such as changes in speech rate, pausing, stress, rhythm or intonation play a major role in motor speech disorders (MSDs), as demonstrated early on in the classification system by Darley, Aronson, and Brown (1969) and many more perceptual and acoustic studies since. Despite their prevalence, relatively few attempts have been made to investigate prosodic impairments in detail compared to segmental aspects of speech production. This is reflected in the clinical field where few standardized prosodic assessment tools or treatment procedures are available.

Amongst the range of prosodic parameters, rate, pause and stress have been investigated most extensively with studies dating back to the 1960s (e.g. Canter, 1963). However, much less information is available on other aspects such as intonation. Most previous research has demonstrated impairments in global aspects such as range and variability of F0. Only a small number of studies have investigated F0 in a more functional way, such as reporting on F0 movements in interrogative-declarative sentence pairs (Le Dorze, Ouellet, \& Ryalls, 1994; Ma, Whitehill, \& So, 2010; Patel, 2002; Penner, Miller, Hertrich, Ackermann, \& Schumm, 2001; Robin, Klouda, \& Hug, 1991) or performance variations across different text styles (Kent \& Rosenbek, 1983; Lowit-Leuschel \& Docherty, 2001). Whilst informative, previous studies are limited by the fact that they primarily measured phonetic aspects of intonation, i.e. F0 variation, but did not consider the phonological, linguistic nature of the pitch movements. As a consequence, it remains unclear whether observed intonational changes are phonological or phonetic in nature, i.e. whether they are the result of differences in the underlying structure of intonation patterns or the way these underlying structures are realized. 


\section{CHARACTERIZING INTONATION DEFICIT IN MSD}

Kent and Kim (2003) see the main reason for the paucity of phonological investigations of intonation in the absence of accepted methods for its characterization. In an attempt to identify alternative ways of describing intonational disturbances, the authors suggest the use of linguistic approaches that proved successful in analyzing intonation in healthy populations such as the autosegmental-metrical (AM) framework (Pierrehumbert, 1980; for an overview see Ladd, 1996). This framework represents a phonological approach to analyzing intonation structures that views them independently from the phonetic features. According to this approach, intonation contours are sequential phonological representations occurring at linguistically meaningful locations. These phonological representations are analysed in terms of sequences of $\mathrm{H}(\mathrm{igh})$ and L(ow) target tones which are categorized into pitch accents and boundary tones depending on their association with either stressed syllables or phrase boundaries.

Although awareness of the potential of the AM framework for the analysis of disordered intonation was already raised a decade ago (Ball \& Rahilly, 2002; Kent \& Kim, 2003; O’Halpin, 2001), it has only been used sporadically in clinical speech research. Investigated disorders include stuttering (Arbisi-Kelm, 2006), Autism Spectrum Disorders (Green \& Tobin, 2009), hypokinetic dysarthria due to Parkinson's Disease (PD) (Mennen, Schaeffler, Watt, \& Miller, 2008) and foreign accent syndrome (FAS) (Kuschmann, Lowit, Miller, \& Mennen, 2012). Although the number of speakers investigated was small in the studies on dysarthria and FAS, they had a common result in that both clinical groups had the same tonal repertoire available as the control speakers, but showed considerable differences with regard to the implementation of these properties in terms of accentuation and phrasing. In addition, there was some indication that the clinical groups could be differentiated on the basis of the intonation performance, i.e. whilst the PD speakers were found to use fewer pitch 


\section{CHARACTERIZING INTONATION DEFICIT IN MSD}

accents than the control speakers, the participants with FAS exhibited a tendency for overaccentuation.

The above findings suggest that the AM approach has the potential to distinguish disordered from unimpaired speech as well as differentiate speech disorders from each other. It therefore offers a promising approach that can function diagnostically and provide new information on the intonational manifestations of different underlying neuropathologies.

This study builds on Kuschmann et al.'s (2012) and Mennen et al.'s (2008) research by including a higher number of participants and directly comparing a variety of MSDs in order to evaluate to what degree different pathologies can be differentiated by their intonational behaviour. Three distinct speaker groups with motor speech impairment, i.e. hypokinetic dysarthria due to PD, ataxic dysarthria and FAS were investigated for this purpose. These speech disorders were selected on the basis that they have been closely associated with intonational disturbances by previous research but are distinct in their underlying neuropathology. Speakers with hypokinetic dysarthria following PD are prone to reduced intonational variation, often characterized as monopitch and monoloudness (Darley, Aronson, \& Brown, 1969; Ma, Whitehill, \& Cheung, 2010; Skodda, Rinsche, \& Schlegel, 2009). Speakers with ataxic dysarthria due cerebellar degeneration, on the other hand, have been reported to show exaggerated or uncontrolled pitch excursions (Schalling \& Hartelius, 2004; Schalling, Hammarberg, \& Hartelius, 2007). Although FAS is not recognised as a disorder linked to a specific neuropathology, previous research into neurogenic FAS has identified features of dysarthria, apraxia as well as aphasia in these speakers (Miller, Lowit, \& O'Sullivan, 2006), each of which is associated with intonational disturbances in itself. Reported changes in intonation include higher mean pitch (Blumstein, Alexander, Ryalls, Katz, \& Dworetzky, 1987), exaggerated terminal falls (Ingram, McCormack, \& Kennedy, 1992; Moen, 2006), inappropriate pitch excursions on prominent syllables (Avila, González, 


\section{CHARACTERIZING INTONATION DEFICIT IN MSD}

Parcet, \& Belloch, 2004) and difficulties to use intonation to indicate interrogativedeclarative contrasts (Berthier, Ruiz, Massone, Starkstein, \& Leiguarda, 1991; Blumstein et al., 1987).

Of the three groups investigated in this study, one was thus associated with generally reduced intonational behaviour $(\mathrm{PD})$ and two with potentially exaggerated patterns (speakers with ataxia and FAS). In addition, the latter two groups differ significantly in the severity and type of other reported prosodic and segmental problems which raises the question whether they can also be differentiated with a more detailed investigation of their intonational patterns, thus warranting their inclusion in this investigation.

In summary, the current study aimed to evaluate the potential of the AM framework for the analysis of intonation in motor speech disorders, focusing in particular on its ability to distinguish different types of motor speech disorders from each other and from unimpaired speech.

\section{Methods}

\section{Participants}

The intonational analyses of disordered speech were based on existing data from 20 speakers collected as part of other research studies (cf. table 1; more information on participants can be found in Kuschmann et al., 2012; Lowit, Dobinson, Timmins, Howell, \& Kröger, 2010; Lowit, Kuschmann, MacLeod, Schaeffler, \& Mennen, 2010). The current sample included eight participants with hypokinetic dysarthria due to idiopathic Parkinson's Disease (PD), eight with dysarthria due to cerebellar ataxia (AT), and four with foreign accent syndrome (FAS). In addition, speech samples from 10 control speakers $(\mathrm{CON})$ were analyzed (27-76 years, $M=59.2$ years, 6 male, 4 female). They were selected to reflect the age, gender and dialectal background of the clinical group. Two CON speakers were taken from the FAS corpus and four from the AT and PD studies respectively. All participants were 


\section{CHARACTERIZING INTONATION DEFICIT IN MSD}

monolingual speakers of British English, encompassing a number of regional accents (mostly Standard Scottish and Southern British English). Hearing and vision of all participants were normal or corrected-to-normal, and they had adequate cognitive skills to complete the study tasks. Formal and informal assessments further ensured that none of the participants had signs of depression, or a history of neurological and/or speech and language difficulties other than their current problems. The participants with AT and PD were matched for severity on the basis of their intelligibility, which had been established by pools of listeners for the monologue tasks in the original studies. No direct comparison of these data was possible as different rating scales had been used in the original studies, therefore matching was based on broad categories of mild, mild/moderate and moderate intelligibility impairment derived from the scores. The speakers with FAS did not present with intelligibility problems and could thus not be matched to the AT and PD groups on this basis. However, Kuschmann et al. (2012) established speech rate reduction and a range of intonational disturbances in structured speech tasks, which warranted their inclusion in this study.

---table 1 about here---

\section{Speech samples}

The present study is based on spontaneous speech as these samples are generally accepted to reflect more natural speech processes than scripted speech. Analysis of such data is thus important to gain an accurate picture of the manifestations of a speech disorder. In addition, the earlier studies applying the AM framework in the clinical context of MSDs focused on structured speech tasks. The analysis of spontaneous speech aimed to complement these findings and help answer the question whether the AM approach can deal with more natural speech data.

As the data for the various participant groups were sourced from different existing research studies, the nature of the spontaneous speech samples collected from each group differed 


\section{CHARACTERIZING INTONATION DEFICIT IN MSD}

slightly. Samples ranged from a monologue describing how to prepare a cup of tea or coffee by the speakers with FAS (Lowit, Miller, \& Poedjianto, 2003; Miller et al., 2007); a description of their last holiday by the speakers with PD; and a retelling of the Cinderella story (Grabe, 2004) by the speakers with AT. Although the sample types thus varied between procedural recall, narrative and story retell, each group was required to recount a familiar tale or process. Previous research with aphasic speakers found no significant differences between these discourse types in relation to measures such as rate, utterance length, occurrence of mazes or information content of output (McNeil et al., 2007; Ulatowska, North, \& MacalusoHaynes, 1981). To further ensure comparability of the current data, statistical analyses were performed to confirm that the current control speakers showed no significant differences between the samples (see reliability section).

For each speech sample, about 30 seconds of speech excluding pauses were analyzed, starting a minimum of 10 seconds into the recording. In addition to these connected speech samples, all speakers had performed a maximum phonation task, which was also included in the current analysis. All speakers were recorded in quiet locations with digital recording equipment. Further details on equipment and procedures for each of the groups can be found in the original study reports.

\section{Transcription procedure}

Intonation was annotated in Praat speech analysis software (version 5.0.11 C)

Boersma \& Weenink, 1992-2012) using the guidelines of the Intonational Variation in English (IViE) transcription system (Grabe 2001), which is based on the AM framework of intonational analysis (Pierrehumbert, 1980). The AM framework served as a basis for the development of a variety of transcription systems, with ToBI (Tones and Break Indices) representing the first intonation transcription to be published for Mainstream American English. Subsequent work on dialects of British English led to the development of IViE. This 


\section{CHARACTERIZING INTONATION DEFICIT IN MSD}

transcription system was adopted for the current study as it allowed the use of a single annotation system for all participants of the present study, who featured a variety of British English dialects (cf. table 1).

Four levels were annotated in order to arrive at the phonological description of the intonation patterns: 1) a word-by-word orthographic transcription, 2) a transcription of phrase boundaries (\%), pauses (\#) and prominent, i.e. phonetically salient, syllables (P) (in terms of prominence no difference was made between stressed and accented syllables), 3) a phonetic transcription of F0 movements on and around the prominent syllables (stressed syllables were marked using capital letters H, M, L (i.e. High, Middle and Low), unstressed and unaccented syllables were indicated by small letters $(h, m, 1)$ ), and 4) a phonological transcription of pitch accents and boundary tones. For the latter, the following structural labels were employed: $\mathrm{H}^{*}$ (high pitch accent), $\mathrm{L}^{*}$ (low pitch accent), $\mathrm{H}^{*} \mathrm{~L}$ (falling pitch accent), ! H*L (downstepped pitch accent), $\mathrm{L} * \mathrm{H}$ (rising pitch accent), $\mathrm{L} * \mathrm{HL}$ (rise-fall pitch accent) and $\mathrm{H}^{*} \mathrm{LH}$ (fall-rise pitch accent). Boundary tones were labeled as $\% \mathrm{~L}$ and L\% (phrase-initial and -final low boundary tone), $\% \mathrm{H}$ and $\mathrm{H} \%$ (phrase-initial and -final high boundary tone) and $\%$ (phrasefinal level boundary tone). The latter label was employed to indicate that the pitch level between the boundary tone and its preceding pitch accent did not change, e.g. $(\mathrm{H}) \%$ denotes a level boundary tone following a high or rising pitch accent. In addition to these traditional IViE labels, the current study used the ToBI labels $\mathrm{X}^{*}$ and $\% \mathrm{X} / \mathrm{X} \%$, which were employed to deal with labeling uncertainties (cf. ToBI annotation guidelines, Beckman \& Ayers-Elam, 1997). $X^{*}$ was used when a syllable could be classified as pitch accented, but the specific type of pitch accent could not be determined; \%X and X\% were employed in cases where the pitch height of the boundary tone could not be unequivocally identified. Figure 1 exemplifies the various transcription levels and labels based on the IViE system for an utterance of a speaker with PD. 


\section{CHARACTERIZING INTONATION DEFICIT IN MSD}

--- figure 1 about here ---

\section{Measures}

In line with Kuschmann et al.'s (2012) and Mennen et al.'s (2008) investigations, a variety of intonation measures were conducted. This included the establishment of an inventory of structural elements, i.e. pitch accents and boundary tones, as well as the prevalence of these elements, i.e. the percentage of occurrence of each pitch accent type in relation to the total number of accents produced by each speaker. Furthermore, the implementation of intonation contours was examined with regard to their phrasing and accentuation patterns. The former was measured in terms of mean length of intonation phrases (IP), the latter was established by measuring the syllable/pitch-accent ratio, which reflects the overall frequency of pitch accentuation. Mean IP length was expressed in the number of syllables produced per IP. The beginning and end of an IP was established following IViE and ToDI guidelines (Transcription of Dutch Intonation; Gussenhoven, Rietveld, Kerkhoff, \& Terken, 2003), according to which IP-boundaries can be marked by pauses, a melodic feature, or the lengthening of pre-boundary syllables. The presence of any of these features, or any combination of these, justified the setting of an IP-boundary. Once IP length had been determined, the syllable/pitch-accent ratio was established by dividing the number of syllables by the number of pitch accents. A higher value is indicative of a lower number of pitch accents per IP.

The analysis of the different intonation aspects was based on a total of 1064 pitch accents and 1319 boundary tones after pitch accents and boundary tones that could not be clearly classified had been excluded (cf. transcription section). The main reason for exclusion of items was poor voice quality and unreliable pitch tracking, which did not allow the crosschecking of perceptual impressions with acoustic information. There was a clear division between the CON speakers and the speech impaired participants in relation to how 


\section{CHARACTERIZING INTONATION DEFICIT IN MSD}

much of the data had to be excluded, i.e. $6 \%$ of pitch accents and $4 \%$ of boundary tones could not be classified in the CON group, whereas an average of $14 \%$ of the pitch accents and $9 \%$ of the boundary tones were ambiguous in the MSD group. This was not surprising given the frequently reported changes to voice quality in people with motor speech disorders.

Analytical problems are recognized in the ToBI transcription approach which has defined the $\mathrm{X} * / \%$ labels specifically for that purpose. However, our data show an attrition rate twice as high as normal in the disordered speaker group. This underlines the importance of gathering sufficient data from pathological speakers to counterbalance such data loss (Kuschmann, Miller, Lowit, \& Mennen, 2011). The current study resulted in on average 80 data points per speaker, which was considerably more than reported in similar previous studies and thus deemed sufficient for evaluation purposes.

In an attempt to relate these intonation measures to potential disturbances at the phonetic level, maximum phonation duration (MPD), speech rate and F0 variability were investigated. MPD was used to assess the influence of phonatory and pulmonary resources on intonation patterns, rate was correlated with the phrasing results, and F0 was considered in relation to the pitch accents produced. In addition, all three measures provided further information on the overall speech performance that would help to differentiate the three clinical groups. The MPD measure was taken from a vowel prolongation task, rate and F0 data were based on the spontaneous speech tasks described above. MPD was expressed in seconds and represents the duration of the fully voiced section of the vowel. Speech rate was expressed in syllables per seconds and was calculated by dividing the total number of syllables per sample by the overall speaking time including pauses. To measure F0 variation, the mean and standard deviation of F0 for the whole speech sample were extracted by a Praat script after assuring that the data samples did not contain measurement errors that could skew 


\section{CHARACTERIZING INTONATION DEFICIT IN MSD}

the results. In order to normalize for the gender related differences in mean F0, variability was expressed as the coefficient of variation (CV F0).

\section{Statistical Analysis}

Given the varied nature of participant groups and the relatively small and unequal sample sizes, non-parametric statistics were used throughout. Group differences were established using the Mann-Whitney-U-Test, and relationships across parameters were established via a Spearman's Rank Correlation coefficient. Significance was determined at $\mathrm{p}=.05$. Where individual MSD groups were compared to control speakers, this related to the whole of the CON group to achieve sufficient statistical power, rather than individually matched pairs.

\section{Reliability}

Intra- and inter-rater agreement for transcription was completed on four speech samples representing each group investigated in the current study, i.e. one CON speaker, and one speaker with PD, AT and FAS, respectively. Agreement rates were sought for intonation phrase (IP) boundaries, prominent syllables (P) and classification of the structural elements, i.e. pitch accents and boundary tones. Intra-rater reliability, conducted by the second author, was high, with $94 \%$ agreement for IP boundaries, $86 \%$ for prominences, and $90 \%$ for the classification of boundary tones and pitch accents. Inter-rater analyses were carried out by a trained speech and language therapist with experience in prosodic transcription following a designated labeling protocol. Agreement for IP boundaries was $76 \%$ and for prominent syllables $92 \%$. Reliability scores for the intonational categories of pitch accents and boundary tones was $82 \%$, matching previously reported inter-rater agreement results for intonation (e.g. Pitrelli, Beckman, \& Hirschberg, 1994).

In addition to inter- and intra-rater reliability measures, it was established whether there were any differences amongst CON speakers in the parameters investigated in this 


\section{CHARACTERIZING INTONATION DEFICIT IN MSD}

paper, in order to ascertain that neither the different regional accents nor the task differences had an effect on speech performance. Mann-Whitney U tests were performed for the parameters of speech rate, $\mathrm{CV}$ F0 and the prevalence of the $\mathrm{H}^{*} \mathrm{~L}$ pitch accent, splitting the group into PD versus AT control speakers to investigate effects of task variance, or into Scottish versus English speakers to control for accent. None of these statistical comparisons approached significance, indicating that results reported below reflect changes in motor control rather than task or dialect specific behaviors.

\section{Results}

\section{Intonation measures}

In relation to the four aspects of intonation investigated, descriptive statistics were used to present the behavioral findings of the different speaker groups. Where appropriate, statistical analyses were conducted using the Mann-Whitney U Test.

\section{Inventory}

Figures 2, 3 and 4 display the types of pitch accents and boundary tones that were used by the different MSD groups and the CON speakers. It is evident that all four groups employed the same pitch accents, namely $H^{*} \mathrm{~L}, ! \mathrm{H}^{*} \mathrm{~L}, \mathrm{~L}^{*} \mathrm{H}, \mathrm{H}^{*}$ and $\mathrm{L}^{*}$. Similar results emerged for boundary tones in phrase-initial position, i.e. all four groups employed \%L and $\% \mathrm{H}$, the two labels that were available to them. In phrase-final position, the high boundary $\mathrm{H} \%$ and level tones $(\mathrm{H}) \%$ and $(\mathrm{L}) \%$ were used by all speaker groups, whereas the low boundary tone $\mathrm{L} \%$ was only part of the inventory of the $\mathrm{CON}$ speakers and the participants with FAS. Overall, the results of the inventorial analysis revealed that all four groups employed the same pitch accents and to a large extent the same boundary tones, indicating that the speakers with MSD had by and large the same structural elements at their disposal as the CON speakers.

--- Figure 2 about here --- 


\section{CHARACTERIZING INTONATION DEFICIT IN MSD}

--- Figure 3 and 4 about here ---

\section{Prevalence}

The analysis of the prevalence of the different pitch accents revealed that in all four speaker groups $\mathrm{H}^{*} \mathrm{~L}$ was the most common pitch accent, followed by $\mathrm{H}^{*}$ (figure 2 ). The pitch accents $! H^{*} \mathrm{~L}$ and $\mathrm{L}^{*}$, were used only rarely. Differences between speaker groups became obvious in the use of $\mathrm{L} * \mathrm{H}$ which was used considerably more often by the speakers with FAS than any of the other groups (FAS - CON: $U=2, z=-2.65, p=.008$; FAS - PD: $U=0, z=-$ $2.72, \mathrm{p}=.006 ;$ FAS - AT: $\mathrm{U}=0, \mathrm{z}=-2.72, \mathrm{p}=.006$ ). Similarly, the AT group produced a greater number of $\mathrm{H}^{*}$ tones than the $\mathrm{CON}$ speakers $(\mathrm{U}=12.5, \mathrm{z}=-2.45, \mathrm{p}=.014)$ and the participants with $\mathrm{PD}(\mathrm{U}=11, \mathrm{z}=-2.21, \mathrm{p}=.027)$. The speakers with AT and FAS thus showed a preference for high or rising patterns compared to the PD and CON groups. A qualitative analysis did not reveal any relationship between these unusual patterns and the location of the accent, or co-occurrence with other accents in the same phrase, and the results could thus not be ascribed to other performance differences noted in these groups that are discussed below.

The prevalence analysis of boundary tones showed that in phrase-initial position $\% \mathrm{H}$ was the most commonly used tone in the CON, PD and AT groups (figure 3). The FAS group showed a relatively balanced use of both initial boundary tones. In comparison to the pitch accent analysis, the speakers with AT and PD performed similarly for this analysis $(\mathrm{U}=16.5$, $\mathrm{z}=-1.63, \mathrm{p}=.103$ ), and both produced a significantly greater amount of high boundary tones than the other two groups (AT - CON: $U=5.5, z=-3.07, p=.002$; AT - FAS: $U=1, z=-$ 2.54, $\mathrm{p}=.011 ; \mathrm{PD}-\mathrm{CON}: \mathrm{U}=17, \mathrm{z}=-2.05, \mathrm{p}=.041 ;$ PD $-\mathrm{FAS}: \mathrm{U}=4, \mathrm{z}=-2.04, \mathrm{p}=.042$ ). The difference between the speakers with FAS and the CON speakers was not significant (U $=11, \mathrm{z}=-1.27, \mathrm{p}=.203)$. 


\section{CHARACTERIZING INTONATION DEFICIT IN MSD}

In phrase-final position, the performances of the speakers with FAS differed again from those of the remaining speaker groups. Whilst most groups largely favored the level boundary tone $(\mathrm{L}) \%$, the speakers with FAS most frequently employed the high boundary tone $\mathrm{H} \%$ (figure 4). This result was again reflected in the statistical results, where the speakers with FAS differed significantly from the other groups in terms of the use of $\mathrm{H} \%$ (FAS - CON: $U=1, z=-2.69, p=.007$; FAS - PD: $U=0, z=-2.72, p=.006$; FAS - AT: $U$ $=0, \mathrm{z}=-2.72, \mathrm{p}=.006)$ and $(\mathrm{L}) \%(\mathrm{FAS}-\mathrm{CON}: \mathrm{U}=2, \mathrm{z}=-2.55, \mathrm{p}=.011$; FAS $-\mathrm{PD}: \mathrm{U}=2$, $\mathrm{z}=-2.38, \mathrm{p}=.017$; FAS - AT: $\mathrm{U}=2, \mathrm{z}=-2.38, \mathrm{p}=.017)$. In addition, the AT group showed a slight tendency towards a greater use of $(\mathrm{H}) \%$ which was reflected in a significant difference to the CON speakers $(\mathrm{U}=16.5, \mathrm{z}=-2.10, \mathrm{p}=.036)$.

In summary, the results of the prevalence analyses revealed comparable performances across groups regarding the most commonly employed pitch accent $\mathrm{H}^{*} \mathrm{~L}$. It also highlighted differences in that the AT and FAS groups showed a propensity towards high or rising patterns. For the boundary tones in phrase-initial position the PD and AT groups used significantly more high boundary tones than the remaining groups, whereas in phrase-final position this was the case for the speakers with FAS.

\section{Phrasing and Accentuation}

Table 2 presents the results of the phrasing patterns for the different speaker groups in terms of intonation phrase (IP) length. The CON speakers produced on average the longest phrases of the four speaker groups, followed by the FAS and PD groups and then the speakers with AT. There was variability among the speaker groups regarding the extent to which IPs were shortened, with some speakers with FAS and PD performing within and others below the range of the CON speakers. None of the speakers with AT reached the performance levels of the CON speakers. The statistical examination confirmed this observation, revealing that the mean phrase length of the CON speakers was significantly 


\section{CHARACTERIZING INTONATION DEFICIT IN MSD}

longer than those of the remaining speaker groups (CON - PD: $U=11.5, z=-2.53, p=.011$; $\mathrm{CON}-\mathrm{AT}: \mathrm{U}=0, \mathrm{z}=-3.55, \mathrm{p}<.0001 ; \mathrm{CON}-\mathrm{FAS}: \mathrm{U}=5, \mathrm{z}=-2.12, \mathrm{p}=.034)$. The PD and FAS groups did not differ significantly from each other $(U=15, z=-.17, p=.865)$, however, the AT group showed a trend to have even shorter IP lengths than the other two clinical groups, although not all comparisons reached significance (AT - PD: $U=14, z=-1.89, p=$ .059 ; AT - FAS: $U=4.5, z=-1.96, p=.050)$.

In relation to the frequency of pitch accentuation, the analysis of the syllable/pitchaccent ratio revealed a higher frequency of pitch-accented words in all three MSD groups than in the CON group (table 2). The CON speakers produced on average one pitch accent every four syllables, whereas the FAS and PD group did so about every 3.5 syllables. The AT group displayed the highest frequency of accentuation, placing a pitch accent every 2.5 syllables. The statistical results confirm that the speakers with AT had a significantly higher frequency of pitch accents than any of the other groups (AT - CON: $U=0, z=-3.56, p<$ .0001 ; AT - PD: $U=0, z=-3.36, p=.001$; AT - FAS: $U=0, z=-2.72, p=.006)$. None of the other group comparisons were significant.

In summary, the analysis of phrasing and accentuation revealed a significantly shorter mean IP length for the MSD groups compared to the CON speakers. In terms of accentuation, the speakers with AT were found to be the only group displaying a significantly higher frequency of pitch accents.

---table 2 about here---

\section{Phonetic Measures}

Table 2 further provides a summary of the mean and SD values for maximum phonation duration (MPD) speech rate and CV F0 per speaker group. Statistical analyses (Mann-Whitney U test) of the MPD show a significantly shorter maximum phonation duration for the speakers with AT compared to the CON speakers $(U=15, z=-2.02, p=$ 


\section{CHARACTERIZING INTONATION DEFICIT IN MSD}

$.043)$ and the speakers with $\operatorname{PD}(U=12, z=-2.1, p=.036)$. None of the other group differences were significant.

The statistical analyses of the speech rate results revealed a significantly slower speech rate for the speakers with AT $(U=2.5, z=-3.34, p=.001)$ and the speakers with FAS $(U=1, z=$ $-2.69, \mathrm{p}=.007)$ compared to the CON speakers. The speakers with AT also differed significantly from the performances of the speakers with $\operatorname{PD}(U=7, z=-2.63, p=.009)$. The remaining group comparisons did not yield significant results.

There was no statistically significant difference in F0 CV across any of the speaker groups, although there was a small trend for the speakers with AT to have a higher level of F0 variability. Two AT speakers performed above and most of the others at the higher end of the normal range. However, there was also one speaker who had a lower F0 CV than the control group. In comparison, the FAS participants performed well within the normal range, as did the majority of the PD speakers, with only two of the eight speakers showing less F0 variation than the control participants.

\section{Correlation of intonation and phonetic measures}

In order to investigate possible links between the observed intonational differences and speech behaviors at the phonetic level, pitch accent prevalence and phrasing results were considered in relation to the phonetic parameters MPD, speech rate and CV F0, where possible through a correlational analysis, or otherwise qualitatively. No statistical analyses were conducted for the speakers with FAS due to the small group size.

Spearman's rank coefficients for MPD and IP length did not yield significant results for any of the participant groups (CON, PD or AT, cf. table 3). On the other hand, IP length, speech rate and the PA-syllable ratio were significantly correlated with each other, but only in the two clinical groups, not in the CON group. The data indicate that participants with slower rates tended to have shorter IPs and produce more pitch accents overall. Qualitative 


\section{CHARACTERIZING INTONATION DEFICIT IN MSD}

analysis of the CV F0 with the prevalence of pitch accents produced showed a generally variable picture. Qualitative consideration of each group showed no particular patterns in the PD group, i.e. the two speakers who had performed below the normal range for CV F0 did not show any difference in pitch accent or boundary tone distribution to the rest of their group or the CON speakers, suggesting that they produced comparable patterns, but with smaller F0 excursions. The FAS group showed equally little relationship between the measures, but differed from the PD participants in that they showed some differences in pitch accent choice with their greater prevalence of $\mathrm{L} * \mathrm{H}$ patterns despite the lack of difference to the CON group in terms of CV F0. Only in the AT group was there an indication of a possible relationship between phonetic and intonation measures, i.e. the speakers performing above or at the top of the normal range for $\mathrm{CV}$ F0 also produced considerably more high pitch accents $\left(\mathrm{H}^{*}\right)$ and high final boundary tones than the rest of their group or the CON speakers. This pattern applied to four of the eight speakers.

--- table 3 about here ---

\section{Discussion}

The aim of this paper was to evaluate the usefulness of the AM approach to chart intonational profiles of different motor speech disorders on the basis of spontaneous speech data, by identifying differences and similarities in their inventory and prevalence of structural elements, phrasing and accentuation patterns.

\section{Inventory}

The inventorial analysis showed that the three MSD groups employed the same pitch accents and to a large extent the same boundary tones as the CON speakers, indicating that they had by and large the same structural elements available as the healthy CON speakers. This finding confirms previous studies on FAS and PD. Verhoeven and Mariën (2010) analyzed conversational data in a woman with FAS and found that she employed the same 


\section{CHARACTERIZING INTONATION DEFICIT IN MSD}

intonation patterns as the CON group. The same was established by Mennen et al. (2008) for read speech of two speakers with Parkinson's induced dysarthria and by Penner et al. (2001) for three speakers with PD. The data are also indirectly supported by a study on lexical tone patterns in Chinese speakers with PD, where researchers found a preserved ability to produce the correct pitch movement despite the presence of monopitch (Ma, 2009). There are no previous studies that have investigated the intonational inventory in ataxic dysarthria, but in view of the strong similarity with the other two MSD groups one could assume that speakers with ataxia are also unlikely to show altered inventories of pitch accents and boundary tones. However, given the small group sizes of the current study, more extensive research is needed to draw firm conclusions as to whether preserved intonational inventories are a norm in MSDs or not.

\section{Prevalence of pitch accents and boundary tones}

Although all four groups had the same range of pitch accents available, they differed in their use of these. Falling pitch accents were the most prevalent pattern in all four groups. However, whilst the CON speakers and PD speakers showed comparable distribution patterns in relation to other pitch accents, the AT and FAS groups differed from this performance by employing a significantly greater number of high and rising pitch accents. In relation to boundary tones, results suggested a higher prevalence of high tones in initial position in the PD and AT groups, and in final position in the FAS group.

The findings for the PD group confirm earlier results by Mennen et al. (2008) who also noted falling pitch accents to be the most common pitch pattern in PD, followed by $\mathrm{H}^{*}$. A less conclusive picture was provided by Penner et al. (2001), who found the prevalence of pitch patterns across their three speakers with PD and their matched CON speakers to be too variable to allow firm group comparisons. The current and Mennen et al.'s (2008) studies thus suggest that speakers with PD are able to make use of the same intonation patterns as the 


\section{CHARACTERIZING INTONATION DEFICIT IN MSD}

CON speakers. This most likely implies that the area of breakdown is at the phonetic level, i.e. impressions of intonational deviations are more likely related to reductions in pitch excursions and overall pitch range (Caekebeke, Jennekens-Schinkel, van der Linden, Buruma, \& Roos, 1991; Ludlow \& Bassich, 1983; Schlenck, Bettrich, \& Willmes, 1993) than choice of pitch accents. This assumption is confirmed in the current study by the fact that the two speakers with PD who performed below the normal range for CV F0 showed no differences in pitch accent distribution to the rest of their group or the CON participants. Further studies relating intonational and phonetic results with perceptual impressions of pitch performance are warranted to confirm this hypothesis.

In contrast to the speakers with PD, the AT group displayed differences in the use of pitch accents with an abnormally high use of $\mathrm{H}^{*}$. Given that no study to date has investigated tonal patterns in ataxic dysarthria, it is unclear to what degree this result can be generalized across all speakers with ataxia. In addition, the current study was unable to determine the reason for this behavior. The higher prevalence of high tones might thus indicate a speaker specific preference that happened to occur in the speakers investigated in the present study. On the other hand it might reflect some form of physiological restriction, which means that simple pitch accents are easier to produce than complex peak and valley combinations. One possible reason for the prevalence of high rather than low tones is strained voice quality characteristic of speakers with AT. Of the three speakers with the highest use of $\mathrm{H}^{*}$ in the current study, two were significantly affected in this regard. The increased vocal fold tension resulting from more forceful phonation and increased effort of speech production could potentially explain the greater prevalence of high tones but data from more speakers are necessary to confirm this fact.

Interestingly, both AT and PD groups showed a greater prevalence of high boundary tones in initial position. Whilst this result is in line with the general pattern of higher tones in 


\section{CHARACTERIZING INTONATION DEFICIT IN MSD}

the AT group, it was unexpected for the speakers with PD who performed similarly to the CON speakers in every other respect. The only other clinical study by Mennen et al. (2008) found a variable pattern in both their $\mathrm{CON}$ and PD speakers regarding initial and final boundary tones. It is thus uncertain to what degree the current results can be generalized to other speakers with PD.

The FAS group showed the highest number of rising pitch accents and final high boundary tones of the four groups. A previous study on another speaker with FAS (Verhoeven \& Mariën, 2010) has also observed this phenomenon and interpreted these intonation contours as continuation markers. That is, these patterns appeared to be employed by the speaker in an attempt to indicate that she had not finished her turn yet and that there was more information to come. The differing use of pitch accents was thus thought to be a compensatory strategy to overcome the communicative issues posed by other restrictions such as short IP lengths in this speaker. Whilst this explanation makes sense when only FAS data are considered, the wider comparison with other types of MSD data raises the question why the PD and particular the AT group, who had the shortest IP length, did not exhibit the same patterns. It thus appears that the rising tones could in fact be an inherent feature of speakers with FAS rather than the result of a compensatory strategy. Alternatively, the AT and PD groups may not have produced continuation markers as a result of other influencing factors. For example, PD speakers might have planned shorter utterances in the first place due to language or cognitive problems, thus not necessitating continuation markers. Again, more controlled experiments are required to investigate this issue further.

\section{Phrasing}

The analysis of IP length revealed that the CON speakers produced significantly longer phrases than any of the three MSD groups. Reduced phrase length is a common feature of motor speech disorders. For ataxic dysarthria, short phrases, i.e. reduced phrase 


\section{CHARACTERIZING INTONATION DEFICIT IN MSD}

length were found by Folker et al. (2010) and Schalling et al. (2007); for speakers with PD this was reported by Cummings, Darkins, Mendez, Hill, and Benson (1988), Grossman et al. (1991), Illes, Metter, Hanson, and Iritani (1988), Mennen et al. (2008) and Tjaden (2009), and for FAS by Wendt, Bose, Scheich, and Ackermann (2007). In addition, a relatively large number of studies on FAS have reported inappropriate inter- and intra word pausing (Berthier et al., 1991; Graff-Radford, Cooper, Colsher, \& Damasio, 1986; Ingram et al., 1992; LauresGore, Contado Henson, Weismer, \& Rambow, 2006; Miller et al., 2006). Given that pauses are one of the main markers of phrasal structuring, the tendency to pause more frequently would result in utterances being divided into smaller phrasing units.

The phonetic results do not provide clear answers for the observed behaviors. In the MPD task only the speakers with AT showed a significantly shorter maximum phonation duration compared to the CON speakers. The PD and AT groups showed significant correlations between IP length and speech rate, with reduced rates resulting in shorter IPs. Results might have been similar for the FAS group but could not be established statistically due to the small group size. The results could suggest that although most disordered speakers had similar breath support available as the CON participants, they took longer to articulate their speech, thus producing fewer words per IP. However, this explanation does not agree with the data for the PD group, who had similar MPD and speech rate values as the CON group, yet still produced shorter IPs. In the absence of clear answers from the current measures, the likely explanation for the observed reduction in IP length in all clinical groups is either that there is a physiological reason that was not picked up by the current task set, or that there is another reason for this behavior. As already alluded to above, shortened IPs might have been due to language or cognitive limitations. Alternatively, they might present a strategy to help the speakers with utterance planning or provide more manageable speech chunks to execute both at a segmental and prosodic level. As before, more controlled 


\section{CHARACTERIZING INTONATION DEFICIT IN MSD}

experiments such as requiring speakers to produce IPs of particular lengths are necessary to investigate this issue further.

\section{Accentuation}

In terms of pitch accentuation the MSD groups all displayed a higher number of pitch accents than the CON speakers. Similar to the phrasing pattern, the speakers with PD and FAS showed comparable performances, which were relatively close to the performances of the CON speakers, whereas the AT group had the highest frequency of accentuation of all groups. Whilst the findings of the speakers with FAS reflect reports from the literature (Kuschmann et al., 2012; Wendt et al., 2007), the higher frequency of accentuation in PD does not align with results by Mennen et al. (2008). They reported a lower number of pitch accents in their two speakers with PD compared to the control speakers, although the difference was relatively small (54 and 55 pitch accents in PD versus 58 and 61 pitch accent in the CON speakers).

In general, the more frequent use of pitch accents observed across a number of participants with MSD is likely to be a consequence of generic rules of intonational wellformedness, which require every phrase to bear at least one pitch accent. Given this relationship between phrasing and accentuation, the increase in pitch accentuation would thus be at least partly related to the fact that many speakers divided their utterances into shorter IPs than the CON speakers. This is confirmed by the significant correlation between the two aspects, with the speakers who produced the shortest phrases as also displaying the highest frequency of pitch accentuation.

\section{Conclusion}

This study has highlighted a number of commonalities and differences in intonational behavior between different types of motor speech disorders and compared to healthy CON speakers applying the AM analysis approach. Clear differences between the speaker groups 


\section{CHARACTERIZING INTONATION DEFICIT IN MSD}

were apparent in three of the four aspects of intonation investigated, i.e. prevalence of intonational elements, phrasing and accentuation. No clear picture emerged that these differences could have been due to the severity of the speech impairment, as captured by perceived intelligibility or acoustic speech measurements. These three parameters thus have potential for charting an intonational profile and for distinguishing different types of motor speech disorders from each other and from healthy speech. The only parameter that turned out not to be informative in terms of identifying group differences was the intonational inventory. Given that all four speaker groups showed the same patterns it appears that the inventory as such might be generally retained in MSDs.

The results evidently have to be interpreted with caution, given the small participant numbers in each group. As already discussed in the various sections above, this study has been able to highlight problem areas, but not necessarily explain why speakers displayed certain impairments. Whilst this study has thus had the advantage of reflecting naturalistic speech behavior, more controlled research will be necessary to pinpoint the exact reasons for this behavior. Such research will be important to further elucidate differences between motor speech disorders and ultimately develop effective treatment strategies for these speakers. In addition, it was not possible to make any assumption on how dysarthria severity affected the current speakers' ability. The original studies providing the current data did not necessarily focus on intonation disturbances and participants had been recruited according to the presence of other speech features. As a consequence, many participants in this study were relatively mildly impaired in relation to intonational disturbances. Although the study successfully identified a range of impairments in all speaker groups, it was unable to inform how these might differ in more severely affected speakers. Future research therefore needs to investigate how the features identified for the current speaker groups compare across 


\section{CHARACTERIZING INTONATION DEFICIT IN MSD}

different levels of severity to arrive at a complete characterization of intonation behavior in the various motor speech disorders.

Despite these shortcomings the current study has demonstrated the value of the AM approach for the characterization of intonational deficits in speakers with MSD. Based on this approach it was possible to differentiate disordered from control speakers, and furthermore, to highlight differences in behavior between speakers with distinct underlying neuropathologies. The AM approach therefore represents a valuable tool, which in combination with phonetic measures has the potential to provide a more precise description of a speaker's intonational impairment, ultimately leading to more effective treatment planning. 


\section{CHARACTERIZING INTONATION DEFICIT IN MSD}

Acknowledgments

This study was supported by the British Academy grant SG-44232, Ataxia UK and

Parkinson's UK grant 8381, as well as a University of Strathclyde Research Development fund. We would also like to thank the participants of the various studies for their time and enthusiasm. 


\section{CHARACTERIZING INTONATION DEFICIT IN MSD}

\section{References}

Arbisi-Kelm, T. R. (2006). An Intonational Analysis of Disfluency Patterns in Stuttering. Doctoral dissertation, University of California, Los Angeles.

Avila, C., González, J., Parcet, M.-A., \& Belloch, V. (2004). Selective alteration of native, but not second language articulation in a patient with foreign accent syndrome. NeuroReport, 15(14), 2267-70.

Ball, M. J., \& Rahilly, J. (2002). Transcribing disordered speech: the segmental and prosodic layers. Clinical Linguistics and Phonetics, 16(5), 329-344.

Beckman, M. E., \& Ayers-Elam, G. (1997). Guidelines for ToBI labeling (version 3, March 1997). The Ohio State University Research Foundation.

Berthier, M. L., Ruiz, A., Massone, M. I., Starkstein, S. E., \& Leiguarda, R. C. (1991). Foreign accent syndrome: behavioral and anatomical findings in recovered and nonrecovered patients. Aphasiology, 5(2), 129-147.

Blumstein, S. E., Alexander, M. P., Ryalls, J. H., Katz, W., \& Dworetzky, B. (1987). On the Nature of the Foreign Accent Syndrome: A Case Study. Brain and Language, 31, $215-244$

Boersma, P., \& Weenink, D. (1992-2012). Praat - doing phonetics by computer. Version 5.0.11 [www.praat.org].

Caekebeke, J. F. V., Jennekens-Schinkel, A., Van der Linden, M. E., Buruma, O. J. S., \& Roos, R. A. C. (1991). The interpretation of dysprosody in patients with Parkinson's disease. Journal of Neurology, Neurosurgery and Psychiatry, 54,145-148.

Canter, G. J. (1963). Speech Characteristics of Patients with Parkinson's disease: Intensity, Pitch and Duration. Journal of Speech and Hearing Disorders, 28(3), 221-229. 


\section{CHARACTERIZING INTONATION DEFICIT IN MSD}

Cummings, J. L., Darkins, A., Mendez, M., Hill, M. A., \& Benson, D. F. (1988). Alzheimer's disease and Parkinson's disease: comparison of speech and language alterations. Neurology, 38, 680-684.

Darley, F. L., Aronson, A. E., \& Brown, J. R. (1969). Differential diagnostic patterns of dysarthria. Journal of Speech and Hearing Research, 12, 246-269.

Folker, J.; Murdoch, B., Cahill, L., Delatycki, M., Corben, L., \& Vogel, A. (2010). Dysarthria in Friedreich's Ataxia: A perceptual analysis. Folia Phoniatrica et Logopaedica, 62, 97-103.

Grabe, E. (2001). The IViE Labelling Guide. Phonetics Laboratory, University of Oxford. Grabe, E. (2004). Intonational variation in urban dialects of English spoken in the British Isles. In P. Gilles \& J. Peters (eds.), Regional Variation in Intonation (pp. 9-31). Tübingen: Niemeyer.

Graff-Radford, N. R., Cooper, W. E., Colsher, P. L., \& Damasio, A. R. (1986). An Unlearned Foreign "Accent" in a Patient with Aphasia. Brain and Language, 28, 86-94.

Green, H., \& Tobin, Y. (2009). Prosodic analysis is difficult ... but worth it: A study in high functioning autism. International Journal of Speech-Language Pathology, 11(4), 308315.

Grossman, M., Carvell, S., Gollomp, S., Stern, M. B., Vernon, G., \& Hurtig, H. I. (1991). Sentence comprehension and praxis deficits in Parkinson's disease. Neurology, 41, $1620-26$.

Gussenhoven, C., Rietveld, T., Kerkhoff, J., \& Terken, J. (2003). ToDI - Transcription of Dutch Intonation. (2nd ed.). University of Nijmegen and University of Eindhoven.

Illes, J., Metter, E. J., Hanson, W. R., \& Iritani, S. (1988). Language production in Parkinson's disease: acoustic and linguistic considerations. Brain and Language, 33, $146-160$. 


\section{CHARACTERIZING INTONATION DEFICIT IN MSD}

Ingram, J. C. L., McCormack, P. F., \& Kennedy, M. (1992). Phonetic analysis of a case of foreign accent syndrome. Journal of Phonetics, 20, 457-474.

Kent, R. D., \& Kim, Y.-J. (2003). Toward an acoustic typology of motor speech disorders. Clinical Linguistics and Phonetics, 17(6), 427-445.

Kent, R. D., \& Rosenbek, J. C. (1983). Acoustic patterns of apraxia of speech. Journal of Speech and Hearing Research, 26, 231-249.

Kuschmann, A., Lowit, A., Miller, N., \& Mennen, I. (2012). Intonation in neurogenic foreign accent syndrome. Journal of Communication Disorders, 45, 1-11.

Kuschmann, A., Miller, N., Lowit, A., \& Mennen, I. (2011). Assessment of Intonation. In: A. Lowit \& R.D. Kent (eds.). Assessment of Motor Speech Disorders. San Diego, C.A.: Plural Publishing Group.

Ladd, D. R. (1996). Intonational phonology. Cambridge: Cambridge University Press.

Laures-Gore, J., Contado Henson, J., Weismer, G., \& Rambow, M. (2006). Two cases of foreign accent syndrome: An acoustic-phonetic description. Clinical Linguistics and Phonetics, 20(10), 781-790.

Le Dorze, G., Ouellet, L., \& Ryalls, J. (1994) Intonation and speech rate in dysarthric speech. Journal of Communication Disorders, 27, 1-18.

Lowit, A., Miller, N., \& Poedjianto, N. (2003). Characteristics of performance change in dysarthria: Clinical perspectives. Journal of Clinical Speech and Language Studies, 12/13, 87-107.

Lowit, A., Dobinson, C., Timmins, C., Howell, P., \& Kröger, B. (2010). The effectiveness of traditional methods and altered auditory feedback in improving speech rate and intelligibility in speakers with Parkinson's disease. International Journal of SpeechLanguage Pathology, 12(5), 426-436. 


\section{CHARACTERIZING INTONATION DEFICIT IN MSD}

Lowit, A., Kuschmann, A., MacLeod, J., Schaeffler, F., \& Mennen, I. (2010). Sentence Stress in Ataxic Dysarthria - A Perceptual Acoustic Study. Journal of Medical SpeechLanguage Pathology, 18(4), 77-82.

Lowit-Leuschel, A., \& Docherty, G. J. (2001). Prosodic variation across sampling tasks in normal and dysarthric speakers. Logopedics Phoniatrics Vocology, 26, 151-164.

Ludlow, C. L., \& Bassich, C. J. (1983). Relationships between perceptual ratings and acoustic measures of hypokinetic speech. In M. R. McNeil, J. C. Rosenbek \& A. E. Aronson (Eds.), The dysarthrias: Physiology acoustics, perception, management. San Diego: College-Hill Press.

Ma, J. K-Y. (2009). Lexical tone production by Cantonese speakers with Parkinson's disease. Proceedings of Interspeech 2009 (pp. 1691 - 1694). Brighton, UK.

Ma, J. K. Y., Whitehill, T., \& Cheung, K. S. K. (2010). Dysprosody and stimulus effects in Cantonese speakers with Parkinson's disease. International Journal of Language and Communication Disorders, 45(6), 645-655.

Ma, J. K-Y., Whitehill, T. L., \& So, S. Y-S. (2010). Intonation contrast in Cantonese speakers with hypokinetic dysarthria associated with Parkinson's disease. Journal of Speech, Language, and Hearing Research, 53(4), 836-849.

McNeil, M. R., Sung, J. E., Yang, D., Pratt, S. R., Fossett, T. R. D., Doyle, P. J., \& Pavelko, S. (2007). Comparing connected language elicitation procedures in persons with aphasia: Concurrent validation of the Story Retell Procedure, Aphasiology, 21(6-8), 775-790.

Mennen, I., Schaeffler, F., Watt, N., \& Miller, N. (2008). An autosegmental-metrical investigation of intonation in people with Parkinson's Disease. Asia Pacific Journal of Speech, Language, and Hearing, 11(4), 205-219. 


\section{CHARACTERIZING INTONATION DEFICIT IN MSD}

Miller, N., Allcock, L., Jones, D., Noble, E., Hildreth, A. J., \& Burn, D. J. (2007). Prevalence and pattern of perceived intelligibility changes in Parkinson's disease. Journal of Neurology, Neurosurgery, and Psychiatry, 78, 1188-1190.

Miller, N., Lowit, A., \& O'Sullivan, H. (2006). What makes acquired foreign accent syndrome foreign? Journal of Neurolinguistics, 19, 385-409.

Moen, I. (2006). Analysis of a case of the foreign accent syndrome in terms of the framework of gestural phonology. Journal of Neurolinguistics, 19, 410-423.

O'Halpin, R. (2001). Intonation issues in the speech of hearing impaired children: analysis, transcription and remediation. Clinical Linguistics and Phonetics, 15(7), 529-550.

Patel, R. (2002). Prosodic Control in Severe Dysarthria. Preserved Ability to Mark the Question-Statement Contrast. Journal of Speech, Language, and Hearing Research, $45,858-870$.

Penner, H., Miller, N., Hertrich, I., Ackermann, H., \& Schumm, F. (2001). Dysprosody in Parkinson's disease: an investigation of intonation patterns. Clinical Linguistics and Phonetics, 15(7), 551-566.

Pierrehumbert, J. (1980). The phonology and phonetics of English intonation. Doctoral dissertation, MIT, Cambridge, MA: MIT Press.

Pitrelli, J., Beckman, M. E., \& Hirschberg, J. (1994). Evaluation of Prosodic Transcription Labelling Reliability in the ToBI framework. Proceedings of the 1994 International Conference on Spoken Language Processing (pp. 123-126). Yokohama, Japan.

Robin, D. A., Klouda, G. V., \& Hug, L. N. (1991). Neurogenic disorders of prosody. In D. Vogel \& M. P. Cannito (eds.), Treating disordered speech motor control: For clinicians by clinicians (pp. 241-271). Austin, Texas: Pro-Ed. 


\section{CHARACTERIZING INTONATION DEFICIT IN MSD}

Schalling, E., \& Hartelius, L. (2004). Acoustic analysis of speech tasks performed by three individuals with spinocerebellar ataxia. Folia Phoniatrica Et Logopaedica, 56, 367380.

Schalling, E., Hammarberg, B., \& Hartelius, L. (2007). Perceptual and acoustic analysis of speech in individuals with spinocerebellar ataxia (SCA). Logopedics Phoniatrics Vocology, 32, 31-46.

Schlenck, K.-J., Bettrich, R., \& Willmes, Z. K. (1993). Aspects of disturbed prosody in dysarthria. Clinical Linguistics and Phonetics, 7, 119-128.

Skodda, S., Rinsche, H., \& Schlegel, U. (2009). Progression of dysprosody in Parkinson's disease over time--a longitudinal study. Movement Disorders, 15(5), 716-722.

Tjaden K. (2009). Speech and swallowing in Parkinson's disease. Topics in Geriatric Rehabilitation, 24, 115-126.

Ulatowska, H. K., North, A. J., \& Macaluso-Haynes, S. (1981). Production of Narrative and Procedural Discourse in Aphasia, Brain and Language, 13, 345-371.

Verhoeven, J., \& Mariën, P. (2010). Neurogenic foreign accent syndrome: Articulatory setting, segments and prosody in a Dutch speaker. Journal of Neurolinguistics, 23(6), 599-614.

Wendt, B., Bose, I., Scheich, H., \& Ackermann, H. (2007). Speech rhythm of a woman with foreign accent syndrome (FAS). In J. Trouvain \& W. J. Barry (eds.), Proceedings of the XVIth International Congress of Phonetic Sciences (pp. 2009-1012). Saarbrücken, Germany. 


\section{CHARACTERIZING INTONATION DEFICIT IN MSD}

Table 1

Information on the participants of the study including age, gender, etiology, dialectal background and intelligibility classification

\begin{tabular}{|c|c|c|c|c|c|}
\hline speaker & age & gender & etiology & dialect & intelligibility \\
\hline PD1 & 71 & $\mathrm{f}$ & IPD & SSBE & mild \\
\hline PD2 & 52 & $\mathrm{~m}$ & IPD & SSBE & mild \\
\hline PD3 & 49 & $\mathrm{f}$ & IPD & SSBE & mild \\
\hline PD4 & 66 & $\mathrm{~m}$ & IPD & SSBE & mild \\
\hline PD5 & 64 & $\mathrm{~m}$ & IPD & SSBE & mild \\
\hline PD6 & 63 & $\mathrm{f}$ & IPD & SSBE & mild/moderate \\
\hline PD7 & 67 & $\mathrm{~m}$ & IPD & SSBE & $\mathrm{mild} /$ moderate \\
\hline PD8 & 69 & $\mathrm{~m}$ & IPD & SSBE & moderate \\
\hline AT1 & 46 & $\mathrm{~m}$ & $\mathrm{CA}$ & SSBE & mild \\
\hline AT2 & 60 & $\mathrm{f}$ & $\mathrm{CA}$ & SSBE & mild \\
\hline AT3 & 52 & $\mathrm{f}$ & $\mathrm{CA}$ & SSBE & mild \\
\hline AT4 & 28 & $\mathrm{f}$ & FA & SSBE & mild \\
\hline AT5 & 65 & $\mathrm{f}$ & SCA6 & SSBE & mild \\
\hline AT6 & 72 & $\mathrm{~m}$ & CA & SSBE & $\mathrm{mild} /$ moderate \\
\hline AT7 & 51 & $\mathrm{~m}$ & $\mathrm{CA}$ & SSBE & $\mathrm{mild} /$ moderate \\
\hline AT8 & 57 & $\mathrm{f}$ & FA & $\mathrm{SSE}$ & moderate \\
\hline FAS1 & 61 & $\mathrm{f}$ & left-hemisphere CVA & SBE (North England) & unimpaired \\
\hline FAS2 & 49 & $\mathrm{f}$ & left-hemisphere CVA & SSE & unimpaired \\
\hline FAS3 & 61 & $\mathrm{~m}$ & brain stem infarct & SSBE & unimpaired \\
\hline FAS4 & 54 & $\mathrm{~m}$ & left-hemisphere CVA & SBE(North England) & unimpaired \\
\hline \multicolumn{6}{|c|}{$\begin{array}{l}\text { Note: } \mathrm{f}=\text { female, } \mathrm{m}=\text { male, IPD - idiopathic Parkinson's Disease, AT - ataxic dysarthria, FAS - foreign accent } \\
\text { syndrome, CA - cerebellar ataxia of undefined type, FA - Friedreich's Ataxia, SCA - Spino-Cerebellar Ataxia } \\
\text { CVA - cerebro-vascular accident, SSBE - Standard Southern British English, SSE - Standard Scottish English } \\
\text { SBE - Standard British English }\end{array}$} \\
\hline
\end{tabular}




\section{CHARACTERIZING INTONATION DEFICIT IN MSD}

Table 2

Overview of the phrasing and accentuation measures, mean IP length (in syllables) and pitch accent-syllable ratio (average distance between PA in syllables) and the phonetic measures, maximum phonation duration (in seconds), speech rate (in syllables per second) and F0 variability (coefficient of variation) per speaker group

\begin{tabular}{cccccc}
\hline & & CON & PD & AT & FAS \\
\hline IP length & mean & 6.96 & 5.51 & 4.24 & 5.63 \\
Syllable - PA ratio & SD & 0.87 & 1.08 & 0.92 & 0.93 \\
& mean & 3.82 & 3.42 & 2.52 & 3.54 \\
MPD & SD & 0.36 & 0.38 & 0.31 & 0.24 \\
& mean & 18.44 & 13.40 & 8.83 & 9.50 \\
speech rate & SD & 10.59 & 4.92 & 4.99 & 3.65 \\
& mean & 3.54 & 3.21 & 2.18 & 2.50 \\
CV F0 & SD & 0.52 & 0.92 & 0.45 & 0.50 \\
& mean & 0.21 & 0.19 & 0.25 & 0.18 \\
& SD & 0.05 & 0.06 & 0.09 & 0.04 \\
\hline
\end{tabular}

Note: $\mathrm{IP}=$ intonation phrase; $\mathrm{PA}=$ pitch accent; $\mathrm{MPD}=$ maximum phonation duration; $\mathrm{CV} \mathrm{F} 0=$ coefficient of variation; $\mathrm{CON}=$ control speakers; $\mathrm{PD}=$ Parkinson's Disease, $\mathrm{AT}=$ ataxic dysarthria, $\mathrm{FAS}=$ foreign accent syndrome 


\section{CHARACTERIZING INTONATION DEFICIT IN MSD}

Table 3

Results for Spearman's Rank correlations between intonation and speech rate measures across speaker groups

\begin{tabular}{|c|c|c|c|c|c|c|c|}
\hline & & \multicolumn{2}{|c|}{ Speech rate } & \multicolumn{2}{|c|}{ IP length } & \multicolumn{2}{|c|}{ PA-syllable ratio } \\
\hline & & $r_{s}$ & $p$ & $r_{s}$ & $p$ & $r_{s}$ & $p$ \\
\hline \multirow[t]{3}{*}{ MPD } & $\mathrm{CON}$ & -.233 & .546 & -.233 & .546 & -.133 & .732 \\
\hline & PD & .119 & .779 & .310 & .456 & .024 & .955 \\
\hline & AT & & .844 & .381 & .352 & .108 & .799 \\
\hline \multirow[t]{3}{*}{ Speech rate } & $\mathrm{CON}$ & & & -.248 & .489 & .248 & .489 \\
\hline & PD & & & .952 & .0001 & .905 & .002 \\
\hline & AT & & & .862 & .006 & .904 & .002 \\
\hline \multirow[t]{3}{*}{ IP length } & $\mathrm{CON}$ & & & & & .430 & .214 \\
\hline & $\mathrm{PD}$ & & & & & .905 & .002 \\
\hline & AT & & & & & .886 & .003 \\
\hline
\end{tabular}




\section{CHARACTERIZING INTONATION DEFICIT IN MSD}

Figure uploaded as a separate document

Figure 1: IViE transcription example showing the four annotation tiers for one of the speakers with PD. Above the tiers the oscillogram (representation of sound wave) as well as the spectrogram (representation of frequency distribution) of the sentence is displayed. The light blue line represents the pitch contour. 


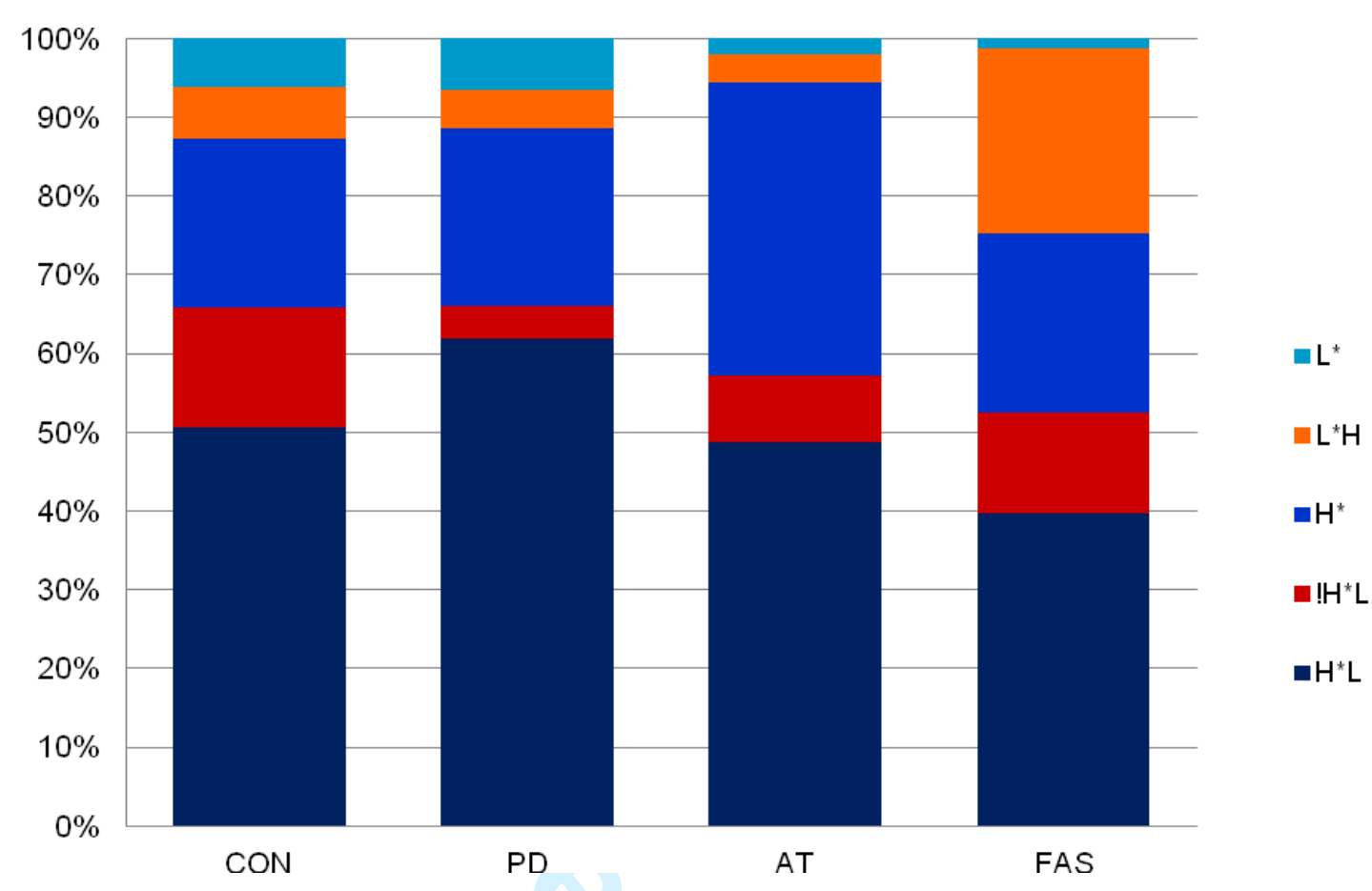

Figure 2: Pitch accent inventory per speaker group in $\%$, i.e. number of total occurrences in $\%(\mathrm{CON}=$ control speakers PD $=$ Parkinson's Disease, $\mathrm{AT}=$ ataxic dysarthria, $\mathrm{FAS}=$ foreign accent syndrome) 


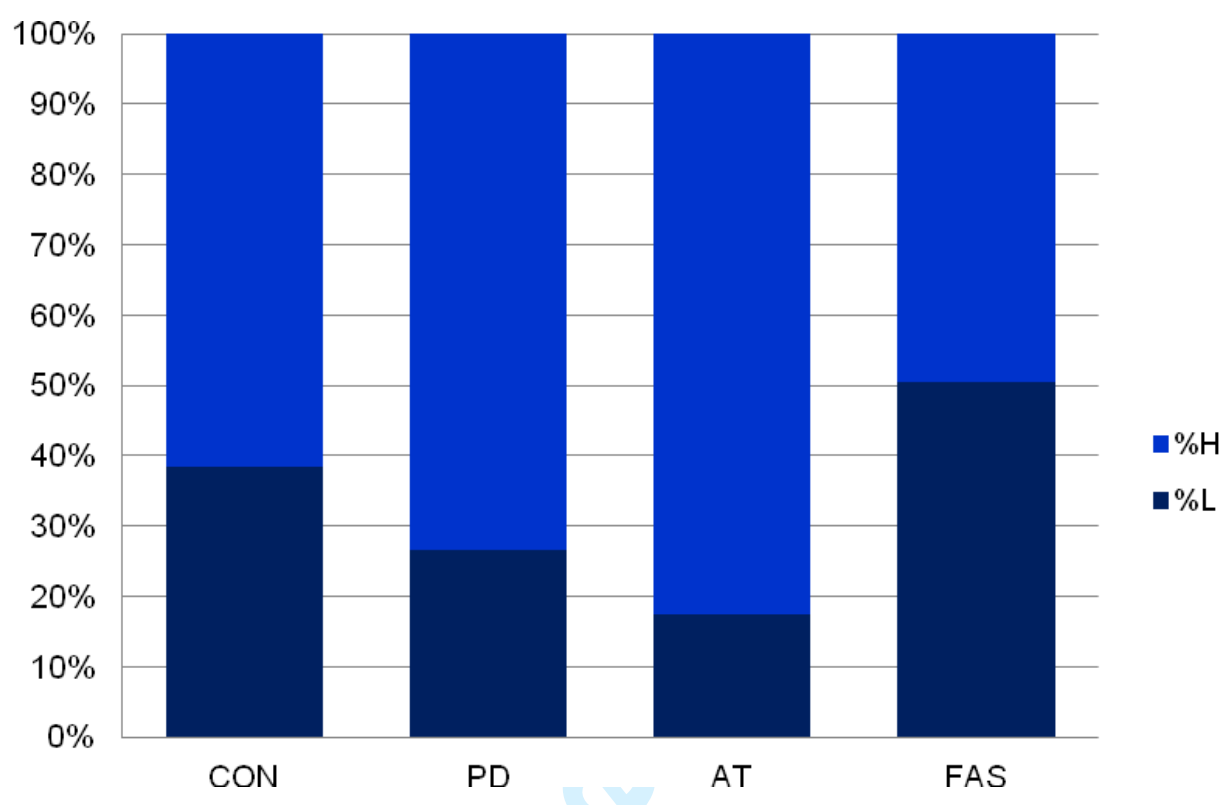

Figure 3: Boundary tone inventory in phrase-initial position per speaker group in $\%(\mathrm{CON}=$ control speakers; $\mathrm{PD}=$ Parkinson's Disease, $\mathrm{AT}=$ ataxic dysarthria, $\mathrm{FAS}=$ foreign accent syndrome) 


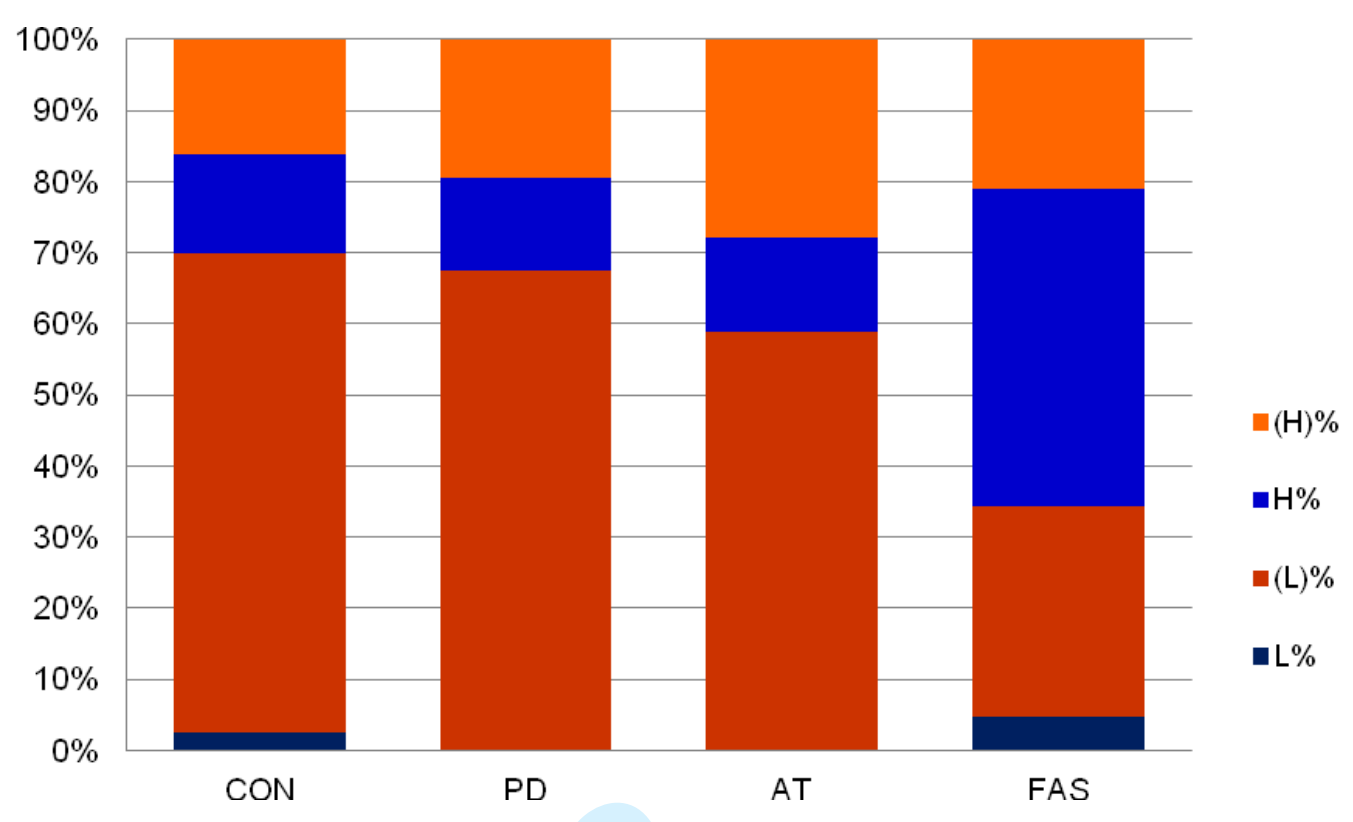

Figure 4: Boundary tone inventory in phrase-final position per speaker group in $\%(\mathrm{CON}=$ control speakers; $\mathrm{PD}=$ Parkinson's Disease, $\mathrm{AT}=$ ataxic dysarthria, FAS = foreign accent syndrome) 


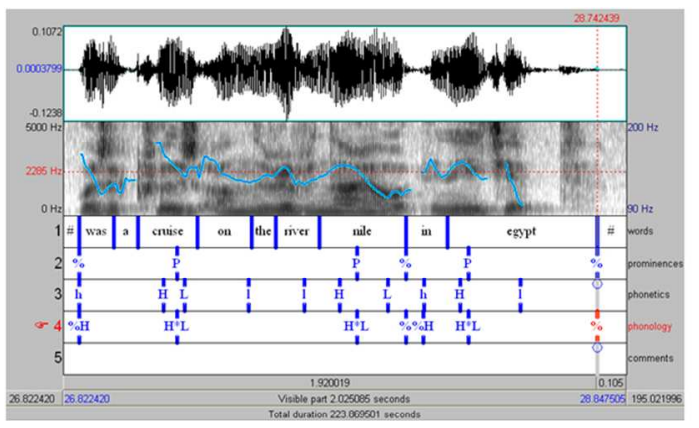

$304 \times 190 \mathrm{~mm}(96 \times 96$ DPI)

30

31

32

33

34

35

36

37

38

39

40

41

42

43

44

45

46

47

48

49

50

51

52

53

54

55

56

57

58

59

60 